\title{
Estudos para construção e propriedades psicométricas da escala contextual de depressão em idosos
}

\author{
Bruno Luis Schaab* \\ Michael Quadros Duarte** \\ Daniel Viana Abs da Cruq***
}

\section{Resumo}

Esta pesquisa teve como objetivo construir uma escala contextual de depressão em idosos baseando-se na importância das intervenções primárias em saúde. Concebeu-se uma versão inicial do instrumento com vinte e sete itens para depressão geriátrica, que foram preenchidos pelo público idoso. A amostra foi constituída por 95 idosos, com idade entre 60 e 83 anos, sendo 22 homens e 73 mulheres. O instrumento construído foi aplicado junto à Escala de Depressão Geriátrica de Yesavage e a Overall Life Satisfaction. Após análises de Curva ROC, quinze itens foram considerados satisfatórios a partir da correlação com o construto de depressão geriátrica e validade discriminante, aferida entre a população com e sem sintomas depressivos. Esses itens passaram pela análise fatorial exploratória, encontrando-se uma solução com quatro fatores e $54 \%$ de variância explicada. O instrumento final apresentou Alpha de Cronbach no valor de 0,77 . Devido a algumas limitações técnicas, sugere-se a continuação do estudo.

Palavras chave: depressão, idosos, escala.

\section{Studies for construction and psychometric properties of the contextual scale of depression in elderly}

\begin{abstract}
This research aimed to build a contextual scale of depression in the elderly, accordingly with the importance of primary interventions in healthcare. A version of the instrument was developed with twenty seven risk factors for geriatric depression that were met by the elderly population. The study sample consisted of 95 elderly subjects, 22 men and 73 women, from 60 to 83 years old. The developed instrument was applied amongst the Yesavage's Geriatric Depression Scale and Overall Life Satisfaction. After statistical analysis, fifteen items were considered satisfactory accordingly with the correlation with the construct of geriatric depression and discriminant validity, measured amongst the population with and without depressive symptoms. These items went through an exploratory factorial analysis, and a solution was found within four factors and 54\% explained variance. The final instrument presented Cronbach's Alpha in the value of 0,77 . Due to some technical limitations, the development of the study is suggested.
\end{abstract}

Key words: depression, elderly, scale

* Graduando em Psicologia da Universidade do Vale do Rio dos Sinos (UNISINOS).

** Graduando em Psicologia da Universidade do Vale do Rio dos Sinos (UNISINOS).

*** Pós-Doutorado em Psicologia Social no Institut de Recerca de Qualitat de Vida da Universitat de Girona (Espanha) e na Universidade Federal do Rio Grande do Sul. Doutorado em Psicologia pela UfRGS, Mestrado em Psicologia Clínica pela UNISINOS, Graduado em Psicologia pela UNISINOS. Atualmente é professor do curso de Psicologia e da Pós-Graduação Lato Sensu da UNISINOS. 


\section{Introdução}

Com o passar dos anos, a população de idosos residentes no Brasil tem crescido consideravelmente. Segundo o que aponta o IBGE (2010), 10,8\% de toda população brasileira era constituída por pessoas acima de 60 anos, com ainda a estimativa de 32 milhões de idosos no ano de 2025. Além do mais, a expectativa de vida de tal camada populacional já contempla, aproximadamente, 76 anos (IBGE, 2016).

Embora a população idosa esteja obtendo maior acesso aos cuidados em saúde e vivendo mais, as características de tal etapa podem interligar-se com o surgimento de diferentes psicopatologias. A depressão e doenças demenciais aparecem como transtornos mais comuns durante o envelhecimento, sendo a primeira mais recorrente (Oliveira, Gomes \& Oliveira, 2006). Além disso, ela tem prevalência estimada de aproximadamente $15 \%$ na população com mais de 60 anos (Cunha, Bastos \& Duca, 2012), com uma taxa de variação da incidência entre 5,4\% e 24,15\% (Pinho, Custódio \& Makdisse, 2009). Trata-se de um fenômeno bastante preocupante durante esta etapa da vida, uma vez que pesquisas têm associado a depressão em idosos a perdas significativas na esfera cognitiva (Lopes, Nascimento, Esteves, Terroso \& Argimon, 2013), ao suicídio (Couto, Prati, Falcão \& Koller, 2008), além de gastos econômicos maiores nos serviços de saúde (Oliveira, Gomes \& Oliveira, 2006).

Uma vez que a população brasileira tem envelhecido, a Psicologia encontra no espaço em questão um campo de diversas possibilidades de intervenção relacionadas a prevenção, promoção e educação em saúde (Neri, 2006). Neste contexto, o Transtorno Depressivo Maior aparece como alvo importante a ser trabalhado. Conforme indicam Santos e Cortina (2011), quando se trata de depressão, a identificação das condições de saúde e as medidas preventivas podem ser exitosas no que diz respeito à promoção do desenvolvimento saudável, além de oferecer menos custos financeiros.

Seguindo tal linha, as intervenções primárias podem ser ações extremamente importantes para a promoção de saúde mental. A Psicologia, historicamente, dirigiu muitas intervenções em relação à psicopatologia segundo o paradigma biomédico, destinando suas práticas ao processo de agir nas manifestações sintomatológicas e comportamentais provenientes da doença por meio da noção de cura, seguindo a lógica de causa e efeito (Delevati \& Souza, 2013). Embora tal modelo possa ser eficiente na perspectiva de saúde e doença, é suposto que o adoecimento psicopatológico segue um curso desenvol- vimental, em que intervenções contextuais podem alterar dinâmicas ambientais ligadas ao surgimento de diferentes psicopatologias.

Dentro de tal contexto, a avaliação psicológica para fins de diagnóstico aparece como função primordial para avaliar a presença ou ausência da sintomatologia depressiva no idoso. Os principais instrumentos utilizados no Brasil tratam-se da Escala de Depressão Geriátrica GDS (na maioria das pesquisas), o Inventário de Depressão de Beck (BDI) e o CES-D (Center for Epidemiological Studies Depression Scale). No entanto, o modo como os referidos instrumentos são construídos levam em conta aspectos sintomatológicos da doença (GDS e CES-D) ou a tríade cognitiva da depressão (BDI). Assim, esses avaliam a possibilidade da presença de depressão, mas não os fatores anteriores que podem estar associados ao seu desfecho. A depressão geriátrica, portanto, é avaliada nesses instrumentos por meio exclusivo de sintomas, sem que se leve em consideração os aspectos sistêmicos e contextuais ligados ao adoecimento.

Segundo Bronfenbrenner (2002), o comportamento do indivíduo em desenvolvimento se estabelece a partir da relação entre diferentes sistemas que se interconectam. Para esse autor, o humano se constitui enquanto entidade em crescimento que, progressivamente, penetra o meio em que reside e o reestrutura. Assim, compreende-se que o adoecimento se dará no contexto, atendendo ao modelo PPCT (pessoa, processo, contexto e tempo) do autor Bronfrenbrenner (2002), no qual se dão as diferentes interações do ser humano. Nos diferentes níveis abordados por esse modelo, irão se situar fatores de risco e proteção ligados ao desenvolvimento (Narvaz \& Koller, 2004). A depressão geriátrica, dessa forma, poderia residir nos comportamentos e recursos do meio que não possibilitam ao indivíduo viver em plenitude e que podem ser caracterizados como fatores de risco ecológicos. Diante disso, o presente trabalho tem por objetivo a construção de uma escala psicométrica para avaliar o contexto de depressão em idosos, tendo por referência a Bioecologia do Desenvolvimento Humano. Para tanto, desenvolveu-se um instrumento contextual, composto de quinze itens para avaliar contextualmente o construto da depressão em idosos.

\section{Método}

\subsection{Construção da escala}

Os itens foram constituídos a partir de fatores de risco para depressão geriátrica encontrados na literatura 
atual. Para tanto, foram identificados 27 fatores distintos que foram distribuídos, tomando por referência o modelo PPCT, entre os diferentes contextos do modelo bioecológico. Posteriormente, tais fatores de risco foram expressos comportamentalmente, formando os itens da escala, que foram analisados quanto a validade aparente por um especialista em avaliação psicológica psicométrica.

Precisamente em relação ao modelo PPCT que subjaz a escala, trata-se de níveis sistêmicos de desenvolvimento humano. Parte-se, inicialmente, da pessoa $(\mathrm{P})$, o que indica um nível mais imediato da experiência pessoal, a partir de componentes biológicos e psicológicos (Poletto \& Koller, 2008). O contexto, por sua vez, é o nível de contato do indivíduo com outros componentes sociais, formando-se pelo microssistema (relação social mais imediata, como a família), o mesossistema (conjunto de microssistemas, como uma escola), o exossistema (níveis sociais que afetam o indivíduo sem que esse participe ativamente) e o macrossistema (cenário político, econômico e cultural da sociedade)(Bronfenbrenner, 1996). O tempo (T) indica que não se pode desassociar o indivíduo do curso temporal, também ligado ao desenvolvimento. Entre tais contextos aconte- cerão os processos, interações recíprocas entre pessoas, símbolos e objetos ligados ao desenvolvimento (Poletto \& Koller, 2008). Na definição do modelo da escala, não foi concebido o exossistema, pelo entendimento de que diversos fatores de risco de outros contextos ecológicos podem ser compreendidos nesse sistema.

\subsection{Delineamento de Pesquisa}

O presente estudo teve a metodologia quantitativa de cunho transversal.

\subsection{Participantes}

Participaram do estudo 95 idosos, com idades compreendidas entre 60 e 83 anos $(\mathrm{M}=69,75 ; \mathrm{DP}=7,44)$, de ambos os sexos, sendo 73 mulheres $(76,8 \%)$ e 22 homens $(23,2 \%)$, a maioria residente na região metropolitana de Porto Alegre (85 idosos) e, em menor escala, dos estados de Minas Gerais e São Paulo (10 idosos). Os participantes localizados fora do Rio Grande do Sul responderam versões online dos instrumentos utilizados, enquanto os moradores da região metropolitana de Porto Alegre utilizaram versões impressas das escalas.

Da referida amostra, 27,4\% dos participantes relataram ter sido diagnosticados com depressão por um pro-

Figura 1 - Modelo teórico da escala contextual de depressão em idosos

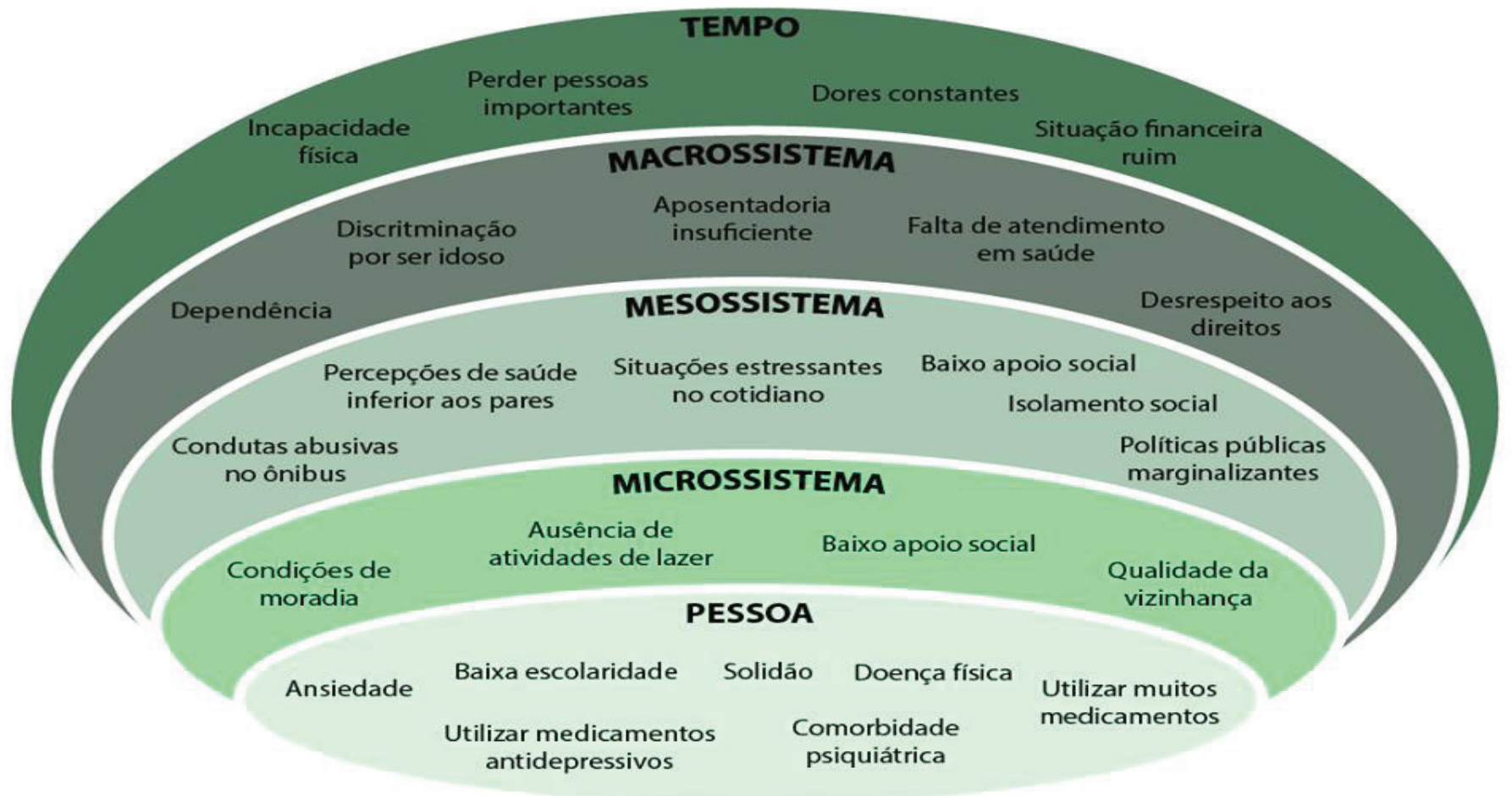


fissional da saúde em algum momento da vida, além de $17,9 \%$ terem na data da coleta de dados o diagnóstico da doença ainda presente. No que diz respeito à formação, 34 participantes tinham o ensino fundamental incompleto $(35,8 \%), 8$ o ensino fundamental completo $(8,4 \%), 9$ o ensino médio incompleto (9,5\%), 27 o ensino médio completo $(28,4 \%), 4$ o ensino superior incompleto $(4,2 \%)$ e $13(13,7 \%)$ o ensino superior completo. A maioria dos idosos recebia a aposentadoria, com um valor a partir do salário-mínimo (94,7\%). Os critérios de inclusão foram: a) ter sessenta anos ou mais b) habilidade de leitura para ler e compreender os itens.

\subsection{Instrumentos}

Os instrumentos utilizados foram: 1) Questionário Sociodemográfico; 2) Escala de depressão contextual para idosos; 3) Escala de depressão geriátrica de 15 itens; 4) Escala OLS - Overall Life Satisfaction.

\subsubsection{Questionário Sociodemográfico}

Desenvolveu-se um questionário sociodemográfico buscando obter dados básicos dos idosos como idade, sexo e formação, além do diagnóstico ou não da depressão por meio de um especialista.

\subsubsection{Escala Contextual de Depressão em Idosos}

A Escala Contextual de Depressão em Idosos (ECDI) foi elaborada a partir da literatura, constituindo-se de 27 itens, pensados a partir de cinco dimensões propostas na Bioecologia do Desenvolvimento Humano, sendo do tipo lickert, pontuável de 0 a 5 .

\subsubsection{Escala de Depressão Geriátrica de Yesavage - versão reduzida (GDS-15)}

A Escala de Depressão Geriátrica, em sua versão reduzida, se trata de um instrumento composto por 15 itens, versando acerca da sintomatologia depressiva entre idosos (Ferrari \& Dalacorte, 2007). O instrumento contém afirmações oriundas dos sintomas de depressão, podendo ser respondidas com um sim ou não, em que se estabelece, respectivamente, uma pontuação binária de 0 ou 1 para cada item (Sousa, Medeiros, Moura, Souza \& Moreira, 2007). Pela soma de todos itens, a pontuação abaixo de cinco é considerada normal, de seis a dez indica depressão que vai da leve a moderada e acima de onze depressão severa (Roesler, Sousa, Ferreira \& Peixoto, 2012).

\subsubsection{Escala de Satisfação Geral com a Vida}

A Overall Life Satisfaction Single Item - OLS é um item escala indicado por Campbell (1976) para estudos sobre o bem-estar. Consiste em um único item que aborda a satisfação com a vida considerada globalmente e é utilizada como medida padrão para estudos sobre qualidade de vida e bem-estar.

\subsection{Procedimentos Éticos}

O projeto foi submetido ao Comitê de Ética em Pesquisa da Universidade X, que aprovou o estudo. Os participantes da pesquisa receberam e assinaram o termo de consentimento livre e esclarecido informando o propósito da pesquisa e indicando os principais regentes éticos, tais quais: possibilidade do idoso poder desistir do estudo a qualquer momento, ter assegurado a confidencialidade dos dados e acesso aos resultados caso desejado, além do contato dos pesquisadores para qualquer dúvida ou desconforto. Na coleta de dados online, o mesmo TCLE foi disponibilizado.

\subsection{Procedimentos de coleta de dados}

A coleta de dados se deu a partir da divulgação da pesquisa em grupos comunitários de idosos na atenção básica de saúde e nos serviços filantrópicos de convivência e fortalecimento de vínculos para tal público. Para tanto, foi agendado um horário, com antecedência, visando aplicar os instrumentos. Alguns idosos, com experiência prévia em tecnologia, participaram da pesquisa respondendo versões online dos instrumentos, a partir da divulgação do estudo em redes sociais.

\subsection{Procedimentos de análise de dados}

Os procedimentos realizados seguiram etapas distintas, contemplando diferentes testes estatísticos. O objetivo inicial foi obter dados descritivos acerca dos participantes do estudo quanto ao diagnóstico de depressão. Em seguida, buscou-se avaliar quais itens do instrumento em desenvolvimento melhor se associavam com o construto de depressão geriátrica (pela Correlação de Pearson), bem como a validade de critério do instrumento aferida pela distinção entre indivíduos com e sem sintomatologia depressiva (pela Curva ROC). Os itens que apresentaram evidências significativas de relação com o construto de depressão geriátrica foram selecionados para compor a versão final do instrumento, totalizando quinze itens, que passaram pela Análise Fatorial Exploratória (AFE). Foram realizadas a Análise Paralela e MAP de Velicer 
para ponderar o número de fatores extraídos, além disso. Esses itens foram, por fim, analisados quanto a sua confiabilidade pelo alfa de Cronbach.

\section{Resultados e discussão}

Entre os participantes do estudo, a sintomatologia depressiva aferida por meio da escala GDS foi $23,2 \%$, dentro da faixa proposta pela literatura (Pinho, Custódio \& Makdisse, 2009).

\subsection{Correlações de Pearson}

No que diz respeito à correlação dos itens com o escore da escala GDS, percebe-se que quinze desses se encontram associados com a depressão geriátrica. Destacam-se os itens "costumo ter alguma doença física" ( $r=0,46$; $\mathrm{p}<0,01)$ e "no decorrer dos anos fiquei incapacitado fisicamente" $(r=0,48 ; p<0,01)$, apresentando os maiores valores de associação. Salienta-se, ainda, que outros itens com viés de adoecimento físico apresentam-se correlacionados, como "utilizo muitos medicamentos" ( $r=0,3$; $\mathrm{p}<0,01)$ e "passei a sentir dores constantes com o passar do tempo" ( $\mathrm{r}=0,29 ; \mathrm{p}<0,01)$. Assim, é perceptível que as questões relacionadas ao adoecimento físico parecem estar intimamente ligadas com o fenômeno na depressão na velhice. A incapacidade física e o adoecimento do corpo aprestam-se fortemente associados com o nível felicidade em idosos, construto esse relacionado com a depressão (Lima, Barros \& Alves, 2012). Além disso, as capacidades físicas parecem ser preponderantes no que tange à depressão geriátrica, haja vista a alta correlação atestada com a incapacidade funcional entre idosos da comunidade (Figueiredo, Pereira, Ferreira, Pereira \& Amorim, 2013) e os altos níveis de associação entre dependência funcional e saúde ruim quando comparados a outras variáveis (Borges, Benedetti, Xavier \& d'Orsi, 2013).

Os quinze itens que apresentaram correlação com o construto de depressão geriátrica foram selecionados para compor a versão final do instrumento no presente estudo. Não é descartável que os itens não correlacionados sejam indicadores de risco, apenas podem não se mostrar adequados à amostra contemplada. Uma das possíveis limitações quanto à correlação é o fato de os participantes serem majoritariamente do sexo feminino. A literatura aponta que o bem-estar subjetivo das mulheres idosas pode ser maior do que o de homens (Cachioni \& Batistoni, 2012).

Por meio da literatura, destaca-se certa tendência de os idosos perceberem sua saúde e bem-estar como positivos, mesmo com situações adversas em seu contexto biopsicossocial, como uso de nicotina, presença de desnutrição, condições ruins de moradia e mesmo sintomatologia depressiva (Silva, Smith-Menezes, Tribess, Rómo-Perez \& Virtuoso Júnior, 2012). Além disso, a Psicologia do Desenvolvimento aponta que os índices de bem-estar subjetivo e felicidade tornam-se maiores na faixa que vai dos 50 aos 85 anos (Papalia \& Feldman, 2013). Supõe-se que muitos itens tenderiam a não se correlacionar com o construto de depressão geriátrica, à medida que poderiam não aumentar ou diminuir seus escores conforme o somatório de itens da escala GDS também variasse. Assim, é possível que mesmo indivíduos acometidos pela depressão possam apresentar escores mais baixos em alguns itens.

Precisamente em relação à satisfação de vida aferida pela OLS, a correlação foi inversa. Dez itens correlacionaram-se com satisfação de vida, sendo que oito dessas já haviam sido igualmente correlacionados com a depressão geriátrica. Apesar de estudos demonstrarem que dimensões relacionadas ao bem-estar apresentam correlações inversas com o construto de depressão (Carneiro, Falcone, Clark, Del Prette \& De Prette, 2007; Dhara \& Jogsan, 2013), deu-se preferência aos itens correlacionados com a GDS e com esta escala para comporem a versão final da ECDI.

Os itens que se mostraram mais associados com a satisfação de vida foram "tenho diversas situações estressantes no meu dia a dia" $(\mathrm{r}=-0,36 ; \mathrm{p}<0,01)$ e "no decorrer dos anos, fiquei incapacitado fisicamente" ( $\mathrm{r}=$ $0,38) ; \mathrm{p}<0,01)$. Assim, para a amostra em questão, parece ser as problemáticas que podem estar relacionadas com menor bem-estar entre idosos. A relação entre o aspecto corpóreo e satisfação de vida aparece bem delineada pela literatura, a partir de estudos que indicam a segunda variável associada à prática de atividade física (Nascimento Júnior, Capelari \& Vieira, 2012). No que tange aos fatores estressores, a satisfação de vida parece ser diretamente proporcional a menor quantidade de eventos que causam estresse no idoso, conforme estudo nacional que verificou tais dimensões entre idosos cuidadores e não cuidadores (Tomomitsu, Perracini \& Neri, 2014).

Analisando a correlação entre os itens da ECDI e o escore fornecido pela GDS, supõe-se uma possível evidência da validade convergente entre aqueles que se mostraram associados. A satisfação de vida, na condição de dimensão psicológica inversa à depressão, também pode ser um indício para validade convergente com os itens da ECDI. Toma-se por base o indicado por Urbina (2007), que aponta as medidas de correlação como um indicador de validade da escala, a partir da noção de convergência de dois instrumentos quanto ao traço latente. 
TABELA 1 - Correlações entre os itens iniciais da ECDI e os escores totais das escalas GDS e OLS

\begin{tabular}{lll}
\hline Item (ECDI) & GDS & OLS \\
\hline 1. Sinto falta de pessoas importantes &, 154 &,- 021 \\
2. Perdi amigos com o passar do tempo &, 103 &, 146 \\
3. Tenho diversas situações estressantes no meu dia a dia &, $331^{*}$ &,$- 365^{*}$ \\
4. Tenho pouco apoio das pessoas &, $279^{*}$ &,- 172 \\
5. Sinto-me satisfeito (a) com o apoio que a sociedade me dá &, 170 & -137 \\
6. Sinto-me isolado (a) do resto da sociedade &, $266^{*}$ &,- 143 \\
7. Sou uma pessoa ansiosa &, $331^{*}$ &,- 202 \\
8. Realizo atividades de lazer em minha comunidade &, $345^{*}$ &,$- 279^{*}$ \\
9. Considero-me uma pessoa de poucos estudos &, 103 & 0,34 \\
10. Utilizo muitos medicamentos &, $306^{*}$ &,- 135 \\
11. O valor da minha aposentadoria é insuficiente &, 056 &,- 079 \\
12. O governo me fornece cuidados ruins em saúde &, 197 &,- 187 \\
13. Costumo ter alguma doença física &, $463 *$ &,- 119 \\
14. Passei a sentir dores constantes com o passar do tempo &, $292^{*}$ &,- 081 \\
15. No decorrer dos anos fiquei incapacitado (a) fisicamente &, $481^{*}$ &,$- 383^{*}$ \\
16. Sofro abusos por ser idoso &, 129 &,- 113 \\
17. Tenho o sentimento de solidão &, $525^{*}$ &,$- 365^{*}$ \\
18. Costumo utilizar medicamento antidepressivo &, $298^{*}$ &,- 156 \\
19. A saúde das pessoas que conheço é melhor que a minha &, $317^{*}$ &,- 135 \\
20. Minha situação financeira era melhor anos atrás &, $280^{*}$ &,$- 221^{*}$ \\
21. Minhas condições de moradia são boas &, 065 &,- 137 \\
22. Gosto dos meus vizinhos &, 173 &,$- 342^{*}$ \\
23. Sou descriminado pela idade &, $347^{*}$ &,$- 226^{*}$ \\
24. Os ônibus que utilizo são bem estruturados para os idosos &, 090 &,$- 267^{*}$ \\
25. Minha saúde mental é boa &, $283^{*}$ &,$- 338^{*}$ \\
26. As leis para idosos me ajudam no dia a dia &, 128 &,- 192 \\
27. Dependo das outras pessoas para viver &, 187 &,- 015 \\
\hline * $<0,01$ & &
\end{tabular}
* $\mathrm{p}<0,01$

\subsection{Curva ROC}

Com o objetivo de certificar a validade de critério do instrumento, dada pela diferenciação dos grupos com e sem sintomatologia depressiva, resultados foram obtidos pela curva ROC, tendo por critério classificatório 6 de 15 pontos no escore da escala GDS. De modo geral, os resultados demonstraram que treze comportamentos descritos na Escala Contextual de Depressão em Idosos tiveram diferenças consideráveis em termos de área e significância estatística, todos anteriormente correlacionados com o construto de depressão geriátrica. A Curva ROC afere a possibilidade de um teste conseguir distinguir duas populações quanto algum critério específico, a partir da área da figura geométrica que se forma entre os eixos de sensibilidade e especificidade de um gráfico, em que quanto mais próximo o valor da área de 1 , maior a possibilidade da curva descriminar dois grupos (Castanho, Barros, Vendite \& Yakamami, 2004).
Compreende-se, no que diz respeito à área, que resultados inferiores a 0,5 não apresentam diferença entre grupos; entre 0,6 e 0,7 apresentam distinção baixa; entre 0,7 e 0,8 , distinção moderada; de 0,8 a 0,9 , distinção boa e; acima de 0,9 ótima distinção. Dos treze comportamentos que conseguiram apresentar diferenças entre participantes com e sem sintomas depressivos, dois estiveram no critério de bom, cinco no critério de regular e seis no critério de baixo. Faz-se ressalva, no entanto, que três itens apresentaram, respectivamente, valores de área de 0,690, 0,695 e 0,695, ficando relativamente próximos dos itens moderados.

Analisando-se os itens, é perceptível que as principais problemáticas que diferenciam indivíduos quanto à presença da sintomatologia depressiva tratam-se de aspectos emocionais negativos, como se sentir ansioso $(A=0,806 ; p<0,000)$ e ter o sentimento de solidão $(A=766 ; p<0,001)$ além de fatores estressantes no cotidia- 
no $(A=0,805 \mathrm{p}<0,000)$. Além disso, é saliente o aspecto corpóreo, marcado por possuir alguma doença física $(A=0,784 ; p<0,000)$ ou ficar incapacitado no decorrer do tempo $(A=0,758 ; p<0,001)$.

Precisamente no que diz respeito a quantidade de itens encontrados como discriminantes, acredita-se que a avaliação da Curva ROC para diferenciar grupos clínicos em termos de fatores de risco contextuais pode apresentar algumas limitações que não se aplicariam a um viés sintomatológico, o que poderia repercutir na Curva ROC. Todo diagnóstico poderá conter uma espécie de erro, isto é, indivíduos doentes identificados como saudáveis e vice-versa. Para tanto, o valor de sensibilidade se dará pela razão entre os indivíduos doentes (verdadeiros positivos) e os doentes que não são diagnosticados (falsos negativos), gerando a seguinte equação: sensibilidade $=$ verdadeiros positivos / (verdadeiros positivos + falsos negativos); a especificidade, por sua vez, será uma razão entre verdadeiros negativos e a soma dos verdadeiros negativos mais os falsos positivos, formando a equação: especifidade $=$ verdadeiros negativos / (verdadeiros negativos + falsos positivos) (Castanho, Barros, Vendite \& Yakamami, 2004). Por esse modelo é notório que um teste sem falsos positivos e falsos negativos apresentará tanto especificidade quanto sensibilidade no valor de 1 .

Pensando pela lógica matemática que sustenta a Curva ROC, é possível imaginar que, tanto para sensibilidade quando especificidade do teste, poderá existir uma grande quantidade de falsos negativos e falsos positivos que influenciam diretamente na área da curva. Em relação aos falsos positivos, há indivíduos que provavelmente foram diagnosticados com depressão pelo instrumento GDS, no entanto apresentam baixos escores nos itens da ECDI, o que faz o valor da especificidade diminuir, essa ligada com a área. Uma das possíveis explicações para tal fenômeno é o fato da depressão poder advir de poucos agentes de risco contextuais - que serão bem pontuados - deixando os demais com valores pequenos. Assim, para o teste, o indivíduo pode parecer um falso negativo, repercutindo diretamente na capacidade discriminante de muitos itens.

No que tange aos falsos negativos, pode ser pensado que outros participantes não foram diagnosticados com depressão pelo instrumento GDS, todavia apresentaram escores altos em itens diversos. Para o teste, esses indivíduos acabam apresentando escores mais elevados quando a tendência seria de escores menores, dado o fato de o idoso não ter depressão pelo critério estabelecido de seis pontos. Mais uma vez, também dada a equação matemática, a sensibilidade do teste poderá ser alterada, baixando o poder discriminante entre grupos quanto aos itens.

Por fim, toma-se por hipótese que alguns itens não puderam apresentar diferença de área pelo fato de terem pontuações similares entre indivíduos com e sem depressão. Por exemplo, considerar a aposentadoria insuficiente e perder amigos com o passar do tempo apresentou médias mais próximas entre o público clínico e não clínico, diferentemente de itens que tratam de se considerar uma pessoa ansiosa ou mesmo ter diversas situações estressantes no dia a dia.

TABELA 2 - Itens descriminados pela análise de curva ROC

\begin{tabular}{lcc}
\hline \multicolumn{1}{c}{ Item da ECD } & Área & Significância \\
\hline 3. Tenho diversas situações estressantes no meu dia a dia &, 805 &, 000 \\
4. Tenho pouco apoio das pessoas &, 695 &, 012 \\
6. Sinto-me isolado do resto da sociedade &, 695 &, 012 \\
7. Sou uma pessoa ansiosa &, 806 &, 000 \\
8. Realizo atividades de lazer em minha comunidade &, 720 &, 005 \\
10. Utilizo muitos medicamentos &, 723 &, 004 \\
13. Costumo ter alguma doença física &, 784 &, 000 \\
14. Passei a sentir dores constantes com o passar do tempo &, 690 &, 015 \\
15. No decorrer dos anos, fiquei incapacitado fisicamente &, 758 &, 001 \\
17. Tenho o sentimento de solidão &, 766 &, 001 \\
18. Costumo utilizar medicamento antidepressivo &, 659 &, 040 \\
20. Minha situação financeira era melhor anos atrás &, 687 &, 016 \\
23. Sou descriminado pela idade &, 679 &, 021 \\
\hline
\end{tabular}


TABELA 3 - Análise fatorial exploratória dos itens correlacionados com a depressão geriátrica

\begin{tabular}{|c|c|c|c|c|}
\hline Itens & Fator 1 & Fator 2 & Fator 3 & Fator 4 \\
\hline 23. Sou descriminado pela idade & ,658 & & & \\
\hline 6. Sinto-me isolado do resto da sociedade & ,644 & & & \\
\hline 20. Minha situação financeira era melhor anos atrás & ,610 & & & \\
\hline 19. A saúde das pessoas que conheço é melhor que a minha & 600 & & & \\
\hline 15. No decorrer dos anos, fiquei incapacitado fisicamente &, 598 & ,400 & & \\
\hline 4. Tenho pouco apoio das pessoas & ,309 & & & \\
\hline 10. Utilizo muitos medicamentos & &, 744 & & \\
\hline $\begin{array}{l}\text { 13. Costumo ter alguma doença física } \\
\text { 18. Costumo utilizar medicamento antidepressivo }\end{array}$ & ,452 & ,661 & & \\
\hline 17. Tenho o sentimento de solidão & ,368 & ,433 & & \\
\hline 14. Passei a sentir dores constantes com o passar do tempo & ,332 & ,399 & & \\
\hline 3. Tenho diversas situações estressantes no meu dia a dia & & & ,851 & \\
\hline 7. Sou uma pessoa ansiosa & &, 576 & ,636 & \\
\hline 8. Realizo atividades de lazer em minha comunidade & & & & ,857 \\
\hline 25. Minha saúde mental é boa & & & &, 570 \\
\hline
\end{tabular}

Elaborada pelos autores, tabela 3, a ser colocada no item 3.3 do artigo

\subsection{Análise Fatorial Exploratória}

Embora tenha sido concebida uma estrutura subjacente para a ECDI, tendo por referência cinco componentes do modelo bioecológico de desenvolvimento proposto por Bronfenbrenner (2002), verificou-se que, para uma solução com cinco fatores, muitas cargas fatoriais apresentaram valores inferiores a 0,3 , o que não é adequado na solução fatorial, segundo Hair, Anderson, Tatham e Black (2005). Por consequência, uma solução com quatro fatores apareceu mais adequada, apresentando cargas satisfatórias entre itens e dimensões, além do fato de todos itens terem contribuído para algum fator. A fim de verificar as hipóteses de soluções fatoriais possíveis, foram realizadas tanto a Análise Paralela, conforme indicação de O'Connor (2000), que apontou 2 fatores como ideais, quanto o Minimum Average Partial Test (MAP) de Velicer, que indicou, conforme a formulação revista dos autores da análise, como sendo unifatorial (O’Connor, 2000). A opção com 2 fatores ou unifatorial, no entanto, não traduz coerência teórica ou lógica entre os itens e os fatores, o que torna razoável e aceitável a solução de 4 fatores adotada.

O índice KMO indicou o valor de 0, 653, demonstrando que análise fatorial estava adequada. A solução com 4 fatores explicou $54 \%$ da variância do instrumento, e a análise contou com comunalidades que oscilaram entre 0,438 e 0,817 , indicando uma boa contribuição dos itens. Como alguns itens carregaram para mais de um fator, optou-se por levar em conta na discussão o fator que obteve maior carga dos itens.

Embora os dois primeiros fatores partilhem cargas em relação a alguns itens, no segundo é evidente, de modo geral, um contexto de limitações na esfera social e perdas que se dão no envelhecimento, abarcando agentes de risco anteriormente concebidos para o mesossistema, macrossistema e cronossistema. O processo de envelhecer é marcado por distintas mudanças frente a situações anteriormente estáveis no âmbito social, cultural, econômico e físico. Assim, os itens "sou discriminado pela idade" (macrossistema) e "sinto-me isolado do resto da sociedade" (mesossistema) aparecem como os mais destacados no fator em questão, indicando situações excludentes tipicamente recorrentes em indivíduos acima de sessenta anos. É notório ainda a presença do cronossistema a partir de itens que demarcam os efeitos da passagem temporal, como "minha situação financeira era melhor anos atrás" e "no decorrer dos anos, fiquei incapacitado fisicamente", além de comportamentos mesossistêmicos que evidenciam a relação com os pares, como "tenho pouco apoio das pessoas" e a "a saúde das pessoas que 
conheço é melhor que a minha”. Em síntese, o presente fator pende para um contexto global de envelhecimento, sobretudo as perdas e mudanças no que diz respeito às relações sociais e culturais, em que o idoso pode sentir-se apartado pela sociedade, malvisto enquanto sujeito e com menos poder financeiro, além de se perceber mais fragilizado fisicamente, mesmo em relação aos pares. Mesmo que os itens não expressem semanticamente a passagem do tempo, é provável que os agentes de risco possam conter tal sentido, haja vista que expressam situações particulares de uma etapa desenvolvimental. Para tanto, ressalta-se a interpenetração e indissociabilidade do modelo PPCT, conforme proposto por Bronfenbrenner (2002).

O segundo fator parece explicitar uma ideia geral de sintomatologia física e mental, ou ainda aspectos patológicos, contando com boa parte dos agentes de risco pensados no nível pessoa, com exceção de um item voltado para o cronossistema, mas com conteúdo altamente ligado com aspectos da experiência individual ("passei a sentir dores constantes com o passar do tempo"). Em nível de saúde mental, encontra-se o item "tenho o sentimento de solidão", o que torna pertinente que o comportamento "costumo utilizar medicamento antidepressivo" apresente-se no mesmo fator, dado sua atuação em tal padrão emocional negativo. Ainda como relevante para o nível pessoa, é destacável o caráter físico e suas repercussões em termos de saúde, contanto com itens como "costumo ter alguma doença física" e "passei a sentir dores constantes com o passar do tempo", tornando relevante $\mathrm{o}$ ato de tomar muitos medicamentos ("utilizo muitos medicamentos"). Tal fator mostra-se pertinente à medida que a saúde mental e física dos idosos estão diretamente ligadas (Mazo, Krug, Virtuoso, Streit \& Benetti, 2012).

O terceiro fator, por sua vez, indica o aspecto da ansiedade no indivíduo idoso, que se mostra relacionado às situações estressantes pelas quais esse passa em seu cotidiano. Deste modo, trata-se de um fator que contempla um item no nível pessoa ("sou uma pessoa ansiosa"), mas também do mesossistema ("tenho diversas situações estressantes no meu dia a dia”). Mais uma vez, verifica-se que diferentes níveis estarão no mesmo fator, o que reforça a ideia de indissociabilidade dos sistemas. A literatura, em especial, aponta a relação direta entre estresse e ansiedade, tanto em idosos quanto em adultos (Margis, Picon, Cosner \& Silveira, 2003).

O quarto fator, por último, apresenta uma ideia de bem-estar subjetivo do idoso, igualmente juntando um item voltado ao nível pessoa ("minha saúde mental é boa") e outro direcionado ao microssistema ("realizo atividades de lazer em minha comunidade"). Assim, torna-se evidente que o ato de realizar determinado exercício de lazer de base comunitária irá se associar com o bem- estar mental do idoso. Estima-se, para tanto, que tais atividades podem colocar o indivíduo em contato com pares - diminuindo o sentimento de solidão - ou mesmo promover a saúde física, à medida que consegue colocar o indivíduo em movimento. Os contatos sociais e grupalidades têm papel significativo na promoção de saúde mental do idoso (Wichmann, Couto, Areosa e Montanes, 2013) e atividade física age no tratamento da depressão em indivíduos acima de sessenta anos (Coelho, Virtuoso Júnior, 2014).

De modo geral, os fatores encontrados não pareceram explicitar a ideia de um sistema propriamente dito embora em alguns casos isso praticamente ocorra -, mas sim de algum fenômeno maior que transpassa os agentes de risco em diferentes contextos. Assim, foi notório o panorama de inserção social do idoso (fator 1), a doença (fator 2) - que se desdobra no âmbito físico e mental -, a ansiedade (fator 3) e bem-estar mental (fator 4). No que diz respeito ao nível individual e social na escala, em suma, apresenta-se bem distinguido por meio dos fatores, haja vista que o primeiro fator abarca a maioria dos aspectos do nível social e o segundo individual, enquanto os demais acabam partilhando ambos os contextos.

No que tange à relação dos resultados encontrados com a estrutura fatorial anteriormente pensada, não foi encontrada uma solução concordante com o estimado. Assim, algumas suposições teóricas podem indicar o porquê de os itens agruparem-se sem seguir a lógica sistêmica pensada:

1. É suposto que o instrumento ECDI trata, a princípio, de fatores particulares e distintos, o que faz com que esses não necessariamente se apresentem como itens de um mesmo sistema ecológico. Por exemplo, dois diferentes fatores de risco podem estar presentes no mesmo sistema, mas não necessariamente possuem uma relação direta, o que faz com que sua covariância seja insignificativa em termos estatísticos e esses não se materializem no mesmo fator.

2. De acordo com Bronfenbrenner (1996), os sistemas se interconectam e confluem. Assim, acredita-se que não seja tão claro diferenciar a posição de cada agente de risco, uma vez que esses não se encontram completamente afastados, mas concatenados e exercendo trocas. Por exemplo, "sentir dores constantes com o passar do tempo" pode indicar, de modo simultâneo, o nível pessoa, mas também a ação do cronossistema. Como indicou a 
análise fatorial, o "isolamento da sociedade" aparece associado ao sentimento de solidão, isto é, percebe-se que diferentes agentes em contextos distintos têm variância conjunta, indicando a indissociabilidade desses.

3. De acordo com Field (2009), os fatores encontrados dizem respeito a estruturas psicológicas maiores compreendidas pelos respondentes. No caso na presente escala, é possível pensar que não está em pauta a ideia de um sistema ou contexto para o idoso, mas sim um fenômeno maior entendido pelos mesmos e que pode abarcar fatores de risco de posições ecológicas distintas.

\subsection{Confiabilidade do Instrumento}

O Alpha de Cronbach do presente instrumento apresentou o valor de 0,77 , o que pode ser considerado satisfatório. É suposto que a escala contou com itens pouco homogêneos, uma vez que se tratam de indicadores de risco distintos, podendo implicar na menor covariância total dos itens.

\section{Considerações finais}

Frente ao fato de a população idosa no Brasil ser alta e a depressão a doença mental mais comum durante tal etapa, além do os instrumentos que mensuram a referida doença se reportarem aos sintomas, o presente estudo visou construir e avaliar propriedades psicométricas de uma escala para diagnosticar o contexto de depressão em idosos. Para tanto, valeu-se do modelo da Bioecologia do Desenvolvimento Humano para compreender como diferentes fatores de risco preconizados pela literatura poderiam interagir e estar no fechamento do adoecimento mental. Assim, o instrumento ECDI foi construído para servir como hipotético indicador com o intento de suscitar intervenções primárias em saúde. Após a realização de distintos procedimentos estatísticos, obteve-se um instrumento com quinze itens e quatro fatores, todavia distintos da estrutura interna prevista.

Analisando o trabalho como um todo, percebe-se que a maioria dos resultados foram satisfatórios frente algumas problemáticas. Uma das limitações do estudo foi em relação amostra, que se deteve a 95 idosos. Observando as análises fatoriais no que diz respeito à literatura, há divergências quanto a quantidade de participantes necessários. Enquanto Hair, Anderson, Tatham e Black (2005) sugerem entre 5 e 10 participantes a cada item, Laros (2004) indica que, para alguns autores, não há nenhum critério demarcando com precisão qual seria a amostra necessária, embora outros cientistas argumentam que é necessário, minimamente, 100 participantes. Embora o índice KMO tenha indicado a adequação da análise fatorial com o valor de 0,653 , além de existirem aproximadamente seis participantes por item, acredita-se que seria mais adequado um número maior de participantes. Problemas como itens que carregam em mais de um fator poderiam ser eliminados ou diminuídos.

Ainda em relação à amostra, deve-se destacar que a mesma não foi heterogênea. O estudo contou com uma população majoritariamente do sexo feminino. Embora seja de conhecimento que a população idosa é maior entre as mulheres, o presente trabalho não conseguiu encontrar resultados similares às distribuições demográficas brasileiras. Além disso, seria importante o estudo contemplar idosos de demais localidades brasileiras, a fim de conseguir uma maior abrangência sociocultural.

No que diz respeito às propriedades psicométricas da escala, a fidedignidade total mostrou valor de Alpha de Cronbach satisfatório. Assim, é possível inferir que a ECDI conseguiu medir bem o que se propõe. Como relatado, é de se esperar que o valor não seja extremamente alto por conta da quantidade de itens existente na escala.

Em relação às evidências de validade de critério, a curva ROC conseguiu sinalizar a diferença de 13 itens entre amostras clínicas e não clínicas, demarcadas pelo escore da GDS. Deste modo, denota-se que, a princípio, o instrumento ECDI pode apresentar uma boa capacidade de distinguir populações. Quando à validade de construto, os mesmos 13 itens e outros 2 se mostraram correlacionados com a depressão geriátrica, totalizando 15 itens.

Em sequência, pretende-se continuar o desenvolvimento do instrumento a partir de uma amostra mais robusta e abrangente. Além disso, serão desenvolvidos critérios para a utilização da escala, visando demarcar pontuações que indicam maior ou menor risco contextual para o desenvolvimento da depressão geriátrica.

\section{Referências}

Borges, L. J., Benedetti, T. R. B., Xavier, A. J., \& d'Orsi, E. (2013). Fatores associados aos sintomas depressivos em idosos: estudo EpiFloripa. Revista de Saúde Pública, 47(4), 701-710.

Bronfenbrenner, U. (1996). A ecologia do desenvolvimento bumano: experimentos naturais e planejados. Porto Alegre: Artes Médicas.

Bronfenbrenner, U. (2002). A ecologia do desenvolvimento bumano: experimentos naturais e planejados. Porto Alegre: Artes Médicas.

Campbell, A. (1976). Subjective measures of well-being. American Psychologist, $31(2), 117$.

Cachioni, M., \& Batistoni, S. S. T. (2012). Bem-estar subjetivo e psicológico na velhice sob a perspectiva do conviver e do aprender. Revista Kairós, 15(14), 09-22.

Carneiro, R. S., Falcone, E., Clark, C., Del Prette, Z., \& Del Prette, A. (2007). Qualidade de vida, apoio social e depressão em idosos: relação com habilidades sociais. Psicologia: Reflexão e Crítica, 20(2), 229-237. 
Castanho, M. J., Barros, L. C., Vendite, L. L., \& Yamakami, A. (2004). Avaliação de um teste em medicina usando uma curva ROC fuzzy. Biomatemática, 14, 19-28.

Coelho, F. G. M., \& Virtuoso Júnior, J. S.(2014). Atividade Física e Saúde Mental do Idoso. Revista Brasileira de Atividade Física e Saúde, 19(5), 663 - 664.

Couto, M. C. P., Prati, L. E., Falcão, D. V. D. S., \& Koller, S. H. (2008). Terapia familiar sistêmica e idosos: contribuições e desafios. Psicologia Clínica, 20(1), 135-152.

Cunha, R. V. D., Bastos, G. A. N., \& Duca, G. F. D. (2012). Prevalence of depression and associated factors in a low income community of Porto Alegre, Rio Grande do Sul. Revista Brasileira de Epidemiologia,15(2), 346-354.

Dhara, R. D., \& Jogsan, Y. A. (2013). Depression and psychological well-being in old age.Journal of Psychology \& Psychotherapy, 3(117), 2161-0487.

Delevati, D. M., \& Souza, A. R. B. (2013). O fazer do psicólogo na saúde. Caderno de Graduação - Ciências Biológicas e da Saúde Fits, 1(2), 79-87.

Ferrari, J. F., \& Dalacorte, R. R. (2007). Uso da Escala de Depressão Geriátrica de Yesavage para avaliar a prevalência de depressão em idosos hospitalizados. Scientia Medica, 17(1), 3-8.

Field, A. (2009). Descobrindo a estatística usando o SPSS. Porto Alegre: Artmed.

Figueiredo, V. F., Pereira, L. S. M., Ferreira, P. H., Pereira, A. M., \& Amorim, J. S. C. (2013). Incapacidade funcional, sintomas depressivos e dor lombar em idosos. Fisioterapia em Movimento, 26(3), 549-557.

INSTITUTO BRASILEIRO DE GEOGRAFIA E ESTATÍSTICA. (2010). Sinopse do Senso Demográfico de 2010. Disponível em: http://www. ibge.gov.br/home/estatistica/populacao/censo2010/default_sinopse.shtm.

INSTITUTO BRASILEIRO DE GEOGRAFIA E ESTATÍSTICA. (2016). Projeção da população do Brasil e das Unidades da Federação. Disponível em: http://www.ibge.gov.br/apps/populacao/projecao/

Laros, J. A. (2004). O uso da análise fatorial: algumas diretrizes para pesquisadores. Em: Pasquali, L. (Org.), Análise fatorial para pesquisadores. Petrópolis: Vozes.

Lima, M. G., Barros, M. B. D. A., \& Alves, M. C. G. P. (2012). Sentimento de felicidade em idosos: uma abordagem epidemiológica,

ISA-Camp 2008. Cadernos de Saúde Pública, 28(12), 2280-2292.

Lopes, R. M. F., Nascimento, R. F. L., Esteves, C. S., Terroso, L. B., \& de Argimon, I. I. L. (2013). Funções executivas de idosos com depressão: um estudo comparativo. Cuadernos de Neuropsicología, 7(2), 72-86.

Margis, R., Picon, P., Cosner, A. F., \& Silveira, R. D. O. (2003). Relação entre estressores, estresse e ansiedade. Revista de Psiquiatria do Rio Grande do Sul, 25(1), 65-74.

Mazo, G. Z., Krug, R. R., Virtuoso, J. F., Streit, I. A., \& Benetti, M. Z. (2012). Autoestima e Depressão em Idosos Praticantes e Exercícios Físicos. Kinesis, 30(1), 188-199.

Narvaz, M. G., \& Koller, S. H.(2004). O modelo bioecológico do desenvolvimento humano. Em: Koller, S. H. (Org.), Ecologia do desenvolvimento humano: Pesquisa e Intervenção no Brasil. São Paulo: Casa do Psicólogo.
Nascimento Júnior, J. R. A. D., Capelari, J. B., \& Vieira, L. F. (2012). Impacto da prática de atividade física no estresse percebido e na satisfação de vida de idosos. Revista da Educação Física / UEM, 23(4), 647-654.

Neri, A. L. (2006). Contribuições da psicologia ao estudo e à intervenção no campo da velhice. Revista Brasileira de Ciências do Envelhecimento Humano, 1(1), 69-80.

O'Connor, B. P. (2000). SPSS and SAS programs for determining the number of components using parallel analysis and Velicer's MAP test. Behavior Research Methods, Instruments \& Computers, 32(3), 396-402.

Oliveira, D. A. A. P., Gomes, L., \& Oliveira, R. F. (2006). Prevalência de depressão em idosos que freqüentam centros de convivência. Revista de Saúde Pública, 40(4), 734-6.

Pinho, M. X., Custódio, O., \& Makdisse, M. (2009). Incidência de depressão e fatores associados em idosos residentes na comunidade: revisão de literatura. Revista Brasileira de Geriatria Gerontologia, 12(1), 123-40.

Papalia, D. E., \& Feldman, R. D. (2013). Desenvolvimento bumano. Porto Alegre: Artmed.

Poletto, M., \& Koller, S. H. (2008). Contextos ecológicos: promotores de resiliência, fatores de risco e de proteção. Estudos de Psicologia, 25(3), 405-416.

Roesler, E., Sousa, A. R. P., Ferreira, L. B., \& Peixoto, H. M. (2012). Prevalência e fatores associados à depressão entre idosos institucionalizados: subsídio ao cuidado de enfermagem. Revista da Escola de Enfermagem da USP, 46(6), 1387-1393.

Santos, L. M., Cortina, I. (2011). Fatores que contribuem para a depressão no idoso. Revista Enfermagem da Unisa, 12(2), 12-16.

Silva, R. J. D. S., Smith-Menezes, A., Tribess, S., Rómo-Perez, V., \& Virtuoso Júnior, J. S. (2012). Prevalence and factors associated with negative health perception by the Brazilian elderly. Revista Brasileira de Epidemiologia, 15(1), 49-62

Sousa, R. L., Medeiros, J. G. M., Moura, A. D., Souza, C. L. M., \& Moreira, I. F. (2007). Validade e fidedignidade da Escala de Depressão Geriátrica na identificação de idosos deprimidos em um hospital geral.Jornal Brasileiro de Psiquiatria, 56(2), 102-7.

Tomomitsu, M. R. S. V., Perracini, M. R., \& Neri, A. L. (2014). Fatores associados à satisfação com a vida em idosos cuidadores e não cuidadores. Ciência \&o Saúde Coletiva, 19(8), 3429-3440.

Urbina, S. (2007). Fundamentos da Testagem Psicológica. Porto Alegre: Artmed.

Wichmann, F. M. A., Couto, A. N., Areosa, S. V. C., \& Montanes, M. C. M. (2013). Grupos de convivência como suporte ao idoso na melhoria da saúde. Revista Brasileira de Geriatria e Gerontoogia, 16(4), 821-832.

Submetido em: 25-1-2017

Aceito em: 2-6-2017 